\title{
Los nombres de aves en el castellano andino de Chota: un enfoque etnolingüístico
}

\author{
Names of birds in the Andean Spanish of Chota: \\ An Ethnolinguistic Approach
}

\author{
Wilmer Burga Muñoz \\ Universidad Nacional Mayor de San Marcos, Lima, Perú \\ wilmer.burga@unmsm.edu.pe \\ ORCID: https://orcid.org/0000-0002-8913-8385
}

\section{Resumen}

El presente artículo estudia las denominaciones de los nombres de aves del castellano andino de Chota desde un enfoque etnolingüístico. En tal sentido, se busca evidenciar las lenguas originarias y los procesos lingüísticos presentes en la formación de los nombres de estas denominaciones. Asimismo, se evidencia la representación simbólica que estas aves tienen en la cosmovisión de los hablantes de Chota.

Palabras clave Nombres de aves, castellano andino, enfoque etnolingüístico, filiación lingüística, procesos lingüísticos, representación simbólica

\begin{abstract}
This research study the denominations of the names of the birds of the Andean Spanish in Chota from an ethnolinguistic approach. In this sense, it seeks to demonstrate the native languages and the linguistic processes present in the formation of names in these denominations. Likewise, the symbolic representation that these birds have in the world view of the Chota speakers is evidenced.
\end{abstract}

Keywords Birds names, Andean Spanish, ethnolinguistic approach, linguistic affiliation, linguistic process, symbolic representation

Fecha de envío: 13/11/2020 Fecha de aceptación: 3/3/2021 


\section{Introducción}

La provincia de Chota, en el departamento de Cajamarca, es un lugar donde existe la convergencia de varias culturas a través de diversos vestigios arqueológi$\cos ^{1}$, y se evidencia la confluencia de lenguas extintas como el cat, el den, el culle, el yunga, el aimara y el quechua que solo existen en topónimos ${ }^{2}$; sin embargo, el quechua es más evidente como sustrato ${ }^{3}$ dentro del castellano andino en general. En ese sentido, se han realizado varios trabajos en toponimia, pero ninguno que solamente aborde el tema de las denominaciones de aves del lugar objeto de estudio. Por ello, en este trabajo interesa evidenciar las lenguas originarias y procesos lingüísticos que subyacen en la formación de los nombres de aves; por ejemplo, en los casos como mulloshingo, chuquia, liwin y potocha, que son peculiares por el contacto cultural y lingüístico.

En este sentido, uno de los primeros registros acerca de las aves fue realizado por Baltazar Jaime Martínez Compañón y Bujanda, obispo de la diócesis de Trujillo (Tumbes, Piura, Lambayeque, La Libertad, Cajamarca, Amazonas y San Martín), entre los años 1779 y 1788, quien resalta por su afán social, cultural y humanista. En consecuencia, realizó una colección valiosa y colorida de los registros de avifauna del norte del Perú de trascendencia histórica (Macera, Jiménez, y Franke, 1997).

Asimismo, Cerna (2015), en su investigación, plantea como hipótesis que muchos nombres con los que el quechuahablante de Cajamarca designa la realidad están motivados por cuestiones onomatopéyicas. De esta manera, analiza algunos nombres como cukuli, lliwin, pishspi, paca-paca, qarqacha, putilla, tuku, turriche y waychaw, para concluir que todos los términos que tienen un carácter fonosimbólico son motivados. Por lo tanto, son motivados las denominaciones de los animales y naturaleza.

Por último, Cancino (2019) explica que en la formación de nombres del quechua de La Unión interviene procesos morfológicos y fonológicos: derivación afijal y no afijal, reduplicación, lexicalización y composición; y bajamiento vocálico, despalatatización, fricativización y sonorización, respectivamente. Algunos de estos procesos posiblemente también se den en la formación de nombres de aves del castellano andino, lo cual compete estudiar. 


\section{Marco teórico}

\subsection{Etnolingüística: lengua, cultura y etnicidad}

Una de las disciplinas lingüísticas que permite estudiar el lenguaje vinculado a determinados grupos sociales y culturales es la etnolingüística. Para Guizzeti, citado por Casado (1991), el objeto de estudio de la etnolingüística está constituida por las relaciones que se dan entre las "estructuras idiomáticas" y aquello que "los pueblos expresan según sus pautas” (p. 39). En este sentido, la lengua dentro de una comunidad lingüística determinada expresa el bagaje cultural de sus miembros y la experiencia que estos han adquirido. No hay lengua en el mundo que no manifieste una sapiencia de hechos culturales como costumbres, tradiciones, ritos, hábitos, actitudes y creencias. De allí la utilidad de la lengua para la etnicidad al ser considerada fundamental en el origen étnico de un grupo social (Siegel, 2018).

\subsection{Aspectos culturales y lingüísticos en Chota}

La historia cultural y lingüística en el área de Chota, hasta donde se dispone, evidencia la presencia o el contacto de diferentes culturas como Pacopampa, Chavín, Chetilla, Wari, Mochica, Huambos e Inca en diferentes etapas de la época precolombina hasta antes de la conquista española en 1532 (Rosas y Shady, 2017; Alarcón, 1991-1992; Watanabe, 2001). En este mosaico de contacto cultural no solo existen vestigios arqueológicos como evidencia de su presencia en este lugar; sino también rastros lingüísticos en el campo de la onomástica, lo que permite explicar el léxico que no corresponde a la lengua materna española de los actuales habitantes. Se han encontrado evidencias de la lengua culle (Andrade-Ciudad, 1999) y su relación con la cultura Chavín (Flores, 2012); asimismo, existen evidencias en relación con la lengua mochica (De la Carrera, 1644, Middendorff, 1960, citado por Berríos, [1985] 2016); y, evidencias de la lengua quechua que sirvió como medio de dominio por la cultura Inca, pero se remonta al protoquechua en relación con la cultura Wari (Isbell, 2010).

\subsection{Lenguas originarias y castellano andino}

La convergencia histórico-cultural y lingüística conlleva a precisar qué se entiende por lenguas originarias. Por lenguas originarias se entiende "como todas aquellas que son anteriores a la difusión del idioma castellano o español y que se preservan y emplean en el ámbito del territorio nacional" (Ministerio de Educación, 
2018, p. 5). Mientras que, el castellano andino se define como una "subvariedad de este conglomerado dialectal, en la medida en que comparte algunos rasgos con el castellano andino sureño y surcentral, de base quechua y aimara, pero también muestra características propias [...] que tiene su propia lógica y organización" (Andrade-Ciudad, 2016, p. 72).

\section{Metodología}

La investigación es de tipo cualitativo, y la muestra está conformada por 28 colaboradores entre hombres y mujeres mayores de 60 años de la ciudad y de seis centros poblados del distrito de Chota de la provincia del mismo nombre, Cajamarca. El corpus está constituido por un total de 39 ornitónimos recogido de fuentes lexicográficas (Cajachismos. Nuestra laya de hablar [2008] de Homero Bazán Zurita, Cajamarquinismos [1979] de Luis Iberico Mas, Monografía de la provincia de chota [1962] de Alejandro Medina Valderrama, El castellano de Chota: chotanismos [2016] de Marco Carbajal Atencio, y Arqueología de Cajamarca: expedición al Marañón, 1937 [2004] de Julio C. Tello) y del estudio de campo mediante una entrevista no estructurada in situ con una grabadora Sony para recopilar información que los hablantes conocen acerca de las aves. Por ello, se ha viajado al lugar objeto de estudio en tres oportunidades, entre 2019 y 2020.

\section{Resultados y discusión}

\subsection{Filiación lingüística de los nombres de la avifauna del castellano andino}

La filiación lingüística de la avifauna de Chota, en el castellano andino, presenta su origen en la lengua quechua de la variedad de Cajamarca Cañaris (Quesada, 1976; Cerna, 2015), mochica (De la Carrera, 1644, Middendorff, 1960, citado por Salas, 2002) y culle (Martínez compañón, citado por Torero, 2002). Así, mediante las fuentes etimológicas y el análisis lingüístico, se ha logrado evidenciar que un grupo de nombres de la avifauna del castellano andino es de origen quechua, como se muestra en la siguiente tabla de ornitónimos. 
Tabla 1.

Nombres de aves de filiación quechua

\begin{tabular}{|l|l|l|l|}
\hline \multicolumn{1}{|c|}{ Ornitónimo } & \multicolumn{1}{|c|}{$\begin{array}{c}\text { Etimología } \\
\text { quechua }\end{array}$} & \multicolumn{1}{|c|}{ Glosa } & Nombre científico \\
\hline 1. Cargacha & qarqacha & 'Pájaro carpintero' & Colaptes rupicola \\
\hline 2. Chuquia & chukyay & 'Zorzal chiguanco' & Turdus chiguanco \\
\hline 3. Guayana & wayanay & 'Golondrina blanquiazul' & Pygochelidon cyanoleuca \\
\hline 4. Huachupishpe & uchu+pishpi & 'Fringilo peruano' & Phrygilus punensis \\
\hline 5. Huaichao & waychaw & 'Arriero de pico negro' & Agriornis montanus \\
\hline 6. Huanchaco & wanchaku & 'Pastorero peruano' & Sturnella belicosa \\
\hline 7. Liclic & liq(i)liq(i) & 'Avefría andina' & Vanellus resplendens \\
\hline 8. Liwin & lliwin & 'Ave no identificada' & Especie no identificada \\
\hline 9. Mulloshingo & mullu + shinku & 'Gallinazo de cabeza & Cathartes aura \\
\hline 10. Pachatuco & pacha + tuku & 'Lechucita peruana' & Glaucidium peruanum \\
\hline 11. Potocha & *putu+ cha & 'Perdiz' & Nothoprocta ornata \\
\hline 12. Quihuela & quiwlla & 'Gaviota andina' & Chroicocephalus serranus \\
\hline 13. Quinde & quinti & 'Picaflor o colibrí' & Boissonneaua mattherwsii \\
\hline 14. Quienquien & kingen/ kinkin & 'Urraca verde' & Cyanocorax yncas \\
\hline 15. Shingo & shinku & $\begin{array}{c}\text { 'Gallinazo de cabeza } \\
\text { negra' }\end{array}$ & Coragyps atratus \\
\hline 17. Suekcha & *uegcha & 'Ave no identificada' & Especie no identificada \\
\hline 18. Tuco & turricha & 'Cucarachero común' & Troglodytes aedon \\
\hline
\end{tabular}

Como se observa, muchos nombres de aves del castellano andino tienen su origen en el quechua, lengua originaria con mayor incidencia lingüística y cultural en Chota. Sin embargo, el castellano andino no solo presenta léxico de filiación quechua, sino también nominaciones que combinan la lengua originaria quechua con la lengua impuesta posteriormente. 
Tabla 2.

Nombres de aves de origen híbrido (quechua-castellano)

\begin{tabular}{|l|l|l|c|}
\hline Ornitónimo & $\begin{array}{l}\text { Etimología. q.- } \\
\text { cast. }\end{array}$ & Glosa & Nombre científico \\
\hline 1. Chinalinda & china + linda & 'Caracara cordillerano' & $\begin{array}{l}\text { Phalcobaenus } \\
\text { megalopterus }\end{array}$ \\
\hline 2. Indiopishgo & indio + pishqu & 'Gorrión de collar rufo' & Zonotrichia capensis \\
\hline
\end{tabular}

Como se observa, los nombres tienen como parte de su etimología a palabras del quechua tal como china 'muchacha' y pishqu 'pájaro'; mientras que 'linda' e 'indio' como nombres del español; por ello, presentan una etimología híbrida.

Asimismo, existen algunas denominaciones de la avifauna de procedencia mochica. En la revisión etimológica de autores como De la Carrera (1644) y Middendorff (1960, citado por Salas, 2002), se han encontrado evidencias para considerar que, en el lugar objeto de estudio, existió también influencia de esta cultura y por ende de su lengua, como se presenta en la siguiente tabla de ornitónimos.

Tabla 3.

Nombres de aves de filiación mochica

\begin{tabular}{|l|c|l|l|}
\hline \multicolumn{1}{|c|}{ Ornitónimo } & $\begin{array}{c}\text { Etimología } \\
\text { mochica }\end{array}$ & Glosa & Nombre científico \\
\hline 1. Chilala & $c h i+l a ́$ & 'Hornero de pata pálida' & Furnarius cinnamomeus \\
\hline 2. Chisco & $c h i s k o$ & 'Sinsonte colilargo' & Mimus longicaudatus \\
\hline 3. Cucula & $c u c u: l i$ & 'Tórtola melódica' & Zenaida meloda \\
\hline
\end{tabular}

De estos ornitónimos, se puede decir que el nombre chilala ha sido asociado al verbo $c h i$ 'estar' y al nombre lá 'agua' del mochica, partiendo del conocimiento de los colaboradores quienes explican que esta ave necesita estar cerca de un manantial de agua o pozo de barro para construir su nido de este material, que se parece a un horno rústico de pan. Asimismo, en cuanto al nombre chisco, ha sido registrado por Middendorf, citado por Salas (2002), como chisko 'tordo'; sin embargo, en el lugar de estudio, encontramos el nombre tordo en alusión a otra ave y chisco en referencia al ave registrada por el autor citado, lo que evidencia el mochiquismo 
en los castellanohablantes. Por último, el nombre cucula está en relación con el peruanismo cuculi y ha sido analizado por Cerrón-Palomino (1989) y Salas (2012) como un nombre de origen mochica.

Otra lengua que presenta evidencia histórica y lingüística, sobre todo en toponimia, es la lengua culle; sin embargo, en la denominación de aves no se ha logrado encontrar evidencias contundentes. No obstante, se ha encontrado el nombre $p i-$ chón del culle pichuñ, que alude a las crías del ave en el español, pero en el lugar objeto de estudio hace alusión a una variedad de aves que alude al indiopishgo, huachupishpe y otras aves sobre todo perjuiciosas. Por ello, a pesar de haber encontrado un solo nombre, se ha considerado dentro del estudio.

En esta indagación etimológica, no todas las denominaciones de las aves evidencian una relación etimológica con una de las lenguas originarias con incidencia histórica y lingüística en el lugar objeto de estudio; más bien, varios de estos nombres presentan un origen desconocido o en rara excepción se puede asociar a otra lengua que no tiene ningún vestigio histórico o lingüístico en el lugar de estudio y no se ha encontrado mayores datos, o en otros casos, algunos de estos nombres son propios del castellano andino.

\section{Tabla 4.}

Nombres de aves de origen desconocido

\begin{tabular}{|l|l|c|}
\hline \multicolumn{1}{|c|}{ Ornitónimo } & \multicolumn{1}{|c|}{ Glosa } & Nombre científico \\
\hline 1. Chiroca & 'Pico grueso de vientre dorado' & Pheucticus chrysogaster \\
\hline 2. Choroco & 'Cucarachero ondeado' & Campylorhynchus fasciatus \\
\hline 3. Garcita & 'Garza bueyera' & Bubulcus ibis \\
\hline 4. Pugo & 'Paloma de puntas blancas' & Leptotila verreauxii \\
\hline 5. Putilla & 'Mosquetero bermellón' & Pyrocephalus rubinus \\
\hline 6. Turca & 'Paloma torcaza' & Patagioenas oenops \\
\hline
\end{tabular}

En relación con los datos, solo el nombre chiroca está en relación con chiroque de la lengua tallán, un antropónimo que significa 'cantor, brabucón' (Cruz-Villegas, 1982), de los pueblos originarios de Piura; asimismo, esta misma ave también es conocida como chiroca en Piura (Puig-Tarrats, 2007) y como chiroque en Lambayeque. Por otra parte, los nombres pugo y choroco son propios del castellano 
andino, y han llegado a lexicalizarse en el área norperuana a partir de las voces onomatopéyicas que emiten estas aves. No obstante, no se puede decir que todos los nombres de aves que derivan de voces onomatopéyicas son propios del castellano andino mediante reglas fonético-fonológicas y de gramaticalización para lexicalizarse, como tuco, huaychao, torricha, cargacha, puesto que muchos de estos nombres tienen su origen en el quechua, lengua que se caracteriza por ser fonosimbólica (Cerna, 2015).

Por último, se ha encontrado un grupo de orinitónimos que proceden del español o castellano, que a su vez tienen su origen en una lengua europea como árabe o latín, como se evidencia en la siguiente tabla.

Tabla 5.

Nombres de aves de filiación español

\begin{tabular}{|l|c|l|c|}
\hline \multicolumn{1}{|c|}{ Ornitónimo } & $\begin{array}{c}\text { Etimología } \\
\text { mochica }\end{array}$ & \multicolumn{1}{|c|}{ Glosa } & Nombre científico \\
\hline 1. Canario & lat. canarius & 'Chirigüe azafranado' & Sicalis flaveola \\
\hline 2. Chotacabras & lat. caprimulgus & 'Chotacabras' & Nyctidromus albicollis \\
\hline 3. Guardacaballos & $\begin{array}{c}\text { germ. warday } \\
\text { lat. caballus }\end{array}$ & $\begin{array}{l}\text { 'Garrapatero de pico } \\
\text { estriado' }\end{array}$ & Crotophaga sulcirostris \\
\hline 4. Halcón & lat.falco-onis & 'Halcón peregrino' & Falco peregrinus \\
\hline 5. Periquito & dim. esp.pero & 'Periquito esmeralda' & Forpus coelestis \\
\hline 6. Patito & ár. hisp.patt & 'Mosquero de agua' & Sayornis nigricans \\
\hline 7. Tordo & lat. turdus & 'Tordo de matorral' & Dives warszewiczi \\
\hline 8. Tortolita & lat. turtur & 'Tortolita peruana' & Columbina cruziana \\
\hline 9. Zorzalito & ár. hisp. zurzál & 'Zorzal grande' & Turdus fuscater \\
\hline
\end{tabular}

En suma, en la denominación de la avifauna de los castellanohablantes de Chota, los nombres de la avifauna se vinculan con las lenguas quechua, mochica y culle, que a su vez tienen incidencia histórica de los pueblos originarios. Asimismo, un grupo menor de denominaciones son de origen desconocido y propias del español o castellano. Por ende, es plausible afirmar aspectos etnolingüísticos léxicos enraizados en los hablantes del castellano andino norperuano, puesto que estos rasgos lingüísticos los hace particulares como grupo social. 


\subsection{Procesos morfológicos en los nombres de la avifauna}

Un mecanismo de formación de los nombres de la avifauna, en el castellano andino, son los nombres compuestos. Este mecanismo es la unión de dos lexemas que al fusionarse forman una sola palabra por yuxtaposición o parasíntesis (Varela, 2018). En este sentido, la formación de nombres de la avifauna de Chota se realiza por yuxtaposición; en esta fusión es evidente la composición de nombre más adjetivo o viceversa, siguiendo la siguiente regla: $\mathrm{N}+\mathrm{ADJ}=\mathrm{N}$ COMP. Asimismo, se tiene el mecanismo de nombre más nombre, siguiendo la regla: $\mathrm{N} 1+\mathrm{N} 2$ = N COMP. Por último, otro mecanismo es la composición mediante verbo más nombre, teniendo como regla: $\mathrm{V}+\mathrm{N}=\mathrm{N}$ COMP. A continuación, la siguiente tabla sintetiza estos mecanismos mencionados.

\section{Tabla 6.}

Formación de nombres de aves por composición

\begin{tabular}{|c|c|}
\hline Composición nombre + adjetivo $=\mathrm{N}$ COMP & Nombre científico \\
\hline \multirow{3}{*}{$\begin{array}{l}\text { china }(N)+\text { linda }(A D J)=\text { Chinalinda } \\
\text { indio }(A D J)+\text { pishgo }(N)=\text { Indiopishgo } \\
\text { mullo }(A D J)+\text { shingo }(N)=\text { Mulloshingo }\end{array}$} & Phalcobaenus megalopterus \\
\hline & Zonotrichia capensis \\
\hline & Cathartes aura \\
\hline Composición nombre + nombre $=$ N COMP & Nombre científico \\
\hline \multirow{2}{*}{$\begin{array}{l}\text { huachu }(\mathrm{N} 1)+\text { pishpe }(\mathrm{N} 2)=\text { Huachupishpe } \\
\text { pacha }(\mathrm{N} 1)+\operatorname{tuco}(\mathrm{N} 2)=\text { Pachatuco }\end{array}$} & Phrygilus punensis \\
\hline & Glaucidium peruanum \\
\hline Composición verbo + nombre $=$ N COMP & Nombre científico \\
\hline \multirow{3}{*}{$\begin{array}{l}\text { chi }(V)+\text { la }(N)+\text { la }(N)=\text { Chilala } \\
\text { chota }(V)+\operatorname{cabras}(N)=\text { Chotacabras } \\
\operatorname{guarda}(V)+\text { caballo }(N)=\text { Guardacaballo }\end{array}$} & Furnarius cinnamomeus \\
\hline & Nyctidromus albicollis \\
\hline & Crotophaga sulcirostris \\
\hline
\end{tabular}

En cuanto a los datos, se evidencia que la palabra chinalinda tiene una combinación que es más regular en castellano al escribir el adjetivo pospuesto al nombre; mientras que en indiopishgo y mulloshingo son más regulares en quechua, ya que el adjetivo está antepuesto al nombre. Asimismo, en los nombres huachupishpe del quechua uchupishpe o de uchupispis 'pájaro que se alimenta de ají' y pachatuco 'ave nocturna' (Quesada, 1976) son una combinación propia del quechua y que 
perdura en el castellano andino. Por último, la palabra chilala tiene origen mochica, aunque no se ha encontrado más evidencias que sigan este proceso de formación morfológica. De igual forma, se tienen dos palabras propias del español: chotacabras y guardacaballo, que siguen una formación de verbo más nombre.

Otro de los mecanismos es la reduplicación, es decir, la repetición de la base, formando una sola palabra (Escandell, 2014). De este modo, los nombres de la avifauna que singuen este mecanismo presentan la siguiente regla: $\mathrm{N} 1+\mathrm{N} 1=$ RED. Esta reduplicación es de carácter onomatopéyico.

\section{Tabla 7.}

Formación de los nombres de aves por reduplicación

\begin{tabular}{|l|c|}
\hline Reduplicación onomatopéyica (N1 + N1 = RED) & Nombre científico \\
\hline Lic (N1) + lic (N1) = Liclic (RED) & Vanellus resplendens \\
\hline Quien (N1) + quien (N1) = Quienquien (RED) & Cyanocorax yncas \\
\hline
\end{tabular}

Asimismo, se ha encontrado un grupo de nombres de la avifauna que se forman mediante la derivación sufijal, cuya formación consiste en agregar sufijos a la base (Varela,2018). En tal sentido, la formación de nombres de las aves de Chota sigue las siguientes reglas: $\mathrm{N}+\mathrm{SUF} .=\mathrm{N}$ DER y S. ONOMAT $+\mathrm{SUF}=\mathrm{N}$ DER. De esta manera, el nombre derivado (N DER) al constituirse por un nombre $(\mathrm{N})$ o sonido onomatopéyico (S. ONOMAT) presenta un sufijo (SUF) -ito/a del español y -cha del quechua.

Tabla 8.

Formación de los nombres de aves por derivación

\begin{tabular}{|c|c|}
\hline Formación de palabras mediante derivación & Nombre científico \\
\hline $\operatorname{garc}(\mathrm{N})+-\mathrm{ita}(\mathrm{SUF})=$ Garcita & Bubulcus ibis \\
\hline pat $(\mathrm{N})+-$ ito $(\mathrm{SUF})=$ Patito & Sayornis nigricans \\
\hline periqu $(\mathrm{N})+$-ito $(\mathrm{SUF})=$ Periquito & Forpus coelestis \\
\hline tortol $(\mathrm{N})+-$ ita $(\mathrm{SUF})=$ Tortolita & Columbina cruziana \\
\hline zorzal $(\mathrm{N})+$-ito $(\mathrm{SUF})=$ Zorzalito & Turdus fuscater \\
\hline poto $(\mathrm{N})+-$ cha $(\mathrm{SUF})=$ Potocha & Nothoprocta ornata \\
\hline carga (S. ONOMAT. $)+$-cha $(\mathrm{SUF})=$ Cargacha & Colaptes rupicola \\
\hline sueq $($ S. ONOMAT. $)+-$ cha $($ SUF $)=$ Suekcha & Especie no identificada \\
\hline torri $(\mathrm{S} . \mathrm{ONOMAT})+$.- cha $(\mathrm{SUF})=$ Torricha & Troglodytes aedon \\
\hline
\end{tabular}


Respecto a los datos, ambos diminutivos tienen un rasgo afectivo, aunque en el quechua presenta un matiz despectivo, puesto que los hablantes explican que son aves perjudiciales, ruidosas o de mal agüero.

Por último, se tiene el proceso de lexicalización, mediante reglas fonético-fonológicas, para formar palabras que luego se introducen en el léxico general (U11man,1972; Bueno, 1994). De esta manera, se puede observar que los hablantes no utilizan la onomatopeya tal como la escuchan; más bien, recurren a mecanismos de adecuación fonológica hasta llegar a una lexicalización del sonido onomatopéyico. Siguiendo la propuesta de Bueno (1994), se observan los siguientes cambios hasta llegar al nombre utilizado por los castellanohablantes: $k r g$ (sonido inarticulado) > karga (interpretación lingüística) > karga $+\widehat{t} a$ adecuación fonológica $)>$ kargat $\widehat{f a}$ (lexicalización) > cargat $\widetilde{f a}$ (nombre del ave). Por lo tanto, el primer paso que realiza el hablante es escuchar un sonido inarticulado emitido por el ave, el segundo paso consiste en una interpretación de la onomatopeya, luego una adecuación fonético-fonológica con ciertas adecuaciones silábicas al castellano andino y, por último, la lexicalización.

Tabla 9.

Formación por lexicalización en los nombres de aves

\begin{tabular}{|c|c|c|c|}
\hline $\begin{array}{c}\text { Sonido } \\
\text { onomatopéyico }\end{array}$ & $\begin{array}{c}\text { Sonido } \\
\text { lexicalizado }\end{array}$ & $\begin{array}{c}\text { Nombre en castellano } \\
\text { andino }\end{array}$ & Glosa \\
\hline țilalalalala & țilala & Chilala & "Hornero de pata pálida" \\
\hline$k r g$ & kargatja & Cargacha & 'Pájaro carpintero' \\
\hline tforok & tforoko & Choroco & 'Cucarachero ondeado' \\
\hline $\bar{t}$ fukiak & tfukia & Chuquia & 'Zorzal chiguanco' \\
\hline kukuli & kukuli & Cuculí & 'Tórtola melódica o cuculí \\
\hline wayt $\widehat{f} a w$ & waychao & Huaychao & 'Arriero de pico negro' \\
\hline Aiwin & liwin & Liwín & 'Especie no identificada' \\
\hline liq & liklik & Liclic & 'Avefría andina' \\
\hline рияииии & pugo & Pugo & 'Paloma de puntas blancas \\
\hline$s q$ & suekcha & Suekcha & 'Especie no identificada' \\
\hline qen & quien & Quienquien & 'Urraca verde' \\
\hline turrik & torrit $\bar{a}$ & Torricha & 'Cucarachero común' \\
\hline tukuuu & tuko & Tuco & 'Búho americano' \\
\hline
\end{tabular}


En resumen, los nombres de la avifauna de Chota siguen ciertos mecanismos en su formación morfológica. En este sentido, se puede encontrar palabras compuestas, por reduplicación, derivación y lexicalización en la denominación de las aves del castellano andino de los hablantes de este lugar.

\subsection{Procesos fonológicos en los nombres de la avifauna}

La sonorización es uno de los procesos que se explica en los nombres de la avifauna de Chota, pues este proceso se ha dado dentro del mismo quechua norteño. Las investigaciones explican que "la sonorización de las oclusivas tras nasal se encuentra hoy solamente en los dialectos quechuas de Colombia, Ecuador y del norte del Perú (Cajamarca, Ferreñafe, Chachapoyas y San Martín)” (Itier, 2013, p. 239). En ese sentido, revisando a Quesada (2006), el quechua de Cajamarca presenta 23 fonemas consonánticos ${ }^{4}$, de los cuales los fonemas /b, d, g, f/ se dan en palabras procedentes del español. A su vez, el fonema " $/ g /$ ha sufrido cierto grado de asimilación, y ha servido para reemplazar tanto a /k/ como a /q/ en topónimos de origen quechua que actúan como préstamos en el español de la región: * $q a$ račuku > /garačugu/ *aqukuču > agokuču" (p. 52). Por lo tanto, la sonorización en el quechua de Cajamarca se da por influencia del español y esta se mantiene en el castellano andino en palabras procedentes del quechua. Así, se puede encontrar este proceso en contextos como en la oclusiva uvular sorda /q/ y la oclusiva velar sorda / $\mathrm{k} /$ del quechua al pasar al castellano andino como oclusiva velar sonora /g/. Asimismo, en el contexto de una oclusiva dental alveolar sorda / $t /$ al pasar a una oclusiva dental alveolar sonora /d/ del castellano andino.

Tabla 10.

La sonorización en los nombres de aves

Sonorización de /q/, /k/y/t/

\begin{tabular}{|c|c|c|}
\hline Lengua originaria & Castellano andino & Glosa \\
\hline 1. /qarqat $\int a /$ & [kargatfa] & 'Pájaro carpintero' \\
\hline 2. /indiopišqu/ & [indiopifgo] & 'Gorrión de collar rufo' \\
\hline 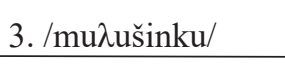 & 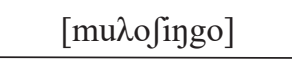 & 'Gallinazo de cabeza roja' \\
\hline 4. /šinku/ & [Jingo] & 'Gallinazo de cabeza negra' \\
\hline 5. /qinti/ & [kinde] & 'Picaflor o colibrî' \\
\hline
\end{tabular}


Las palabras consignadas presentan en su estructura un segmento /q/, /k/ y /t/ tras nasal [n] y vibrante [r], lo cual refuerza la hipótesis de la sonorización dentro del quechua; sin embargo, partiendo del sistema fonológico propuesto por Quesada (2006), esta sonorización se daría por el contacto con el español en el castellano andino. A continuación, la regla que corresponde a este proceso.

Regla: $[$-sonoro $]>[$ +sonoro $] /[$ sonante $]$

El segundo proceso que se evidencia en las denominaciones de la avifauna de Chota es el bajamiento vocálico en cuanto a la altura de la vocal; por ello, también se le conoce como descenso vocálico que consiste en "la variación que presentan los fonemas" (Pérez, 2016, p. 119). Los nombres de aves de Chota presentan este proceso fonológico al pasar de una vocal final cerrada /u/ de una lengua originaria hacia una vocal final media /o/ del castellano andino o de una vocal inicial cerra$\mathrm{da} / \mathrm{i} / \mathrm{a}$ una vocal inicial media /e/ o de una vocal inicial media /e/ a una central abierta $/ \mathrm{a} /$.

\section{Tabla 11.}

Bajamiento vocálico en los nombres de aves

Bajamiento vocálico de /u/, /i/

\begin{tabular}{|c|c|c|}
\hline Lengua originaria & Castellano andino & Glosa \\
\hline 1. /muגušinku/ & 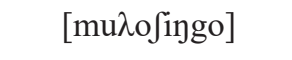 & 'Gallinazo de cabeza roja' \\
\hline 2. /indiopišqu/ & [indiopifgo] & 'Gorrión de collar rufo' \\
\hline 3. /patfatuku/ & [patfatuko] & 'Lechucita peruana' \\
\hline 4. /pitfuñ/ & [pitfón] & 'Especie no identificada' \\
\hline 5. /putu $+\overparen{\mathrm{t} \int \mathrm{a} /}$ & {$\left[\operatorname{potot} \int \mathrm{a}\right]$} & 'Perdiz' \\
\hline 6. /wayt $\widehat{\text { faw/ }}$ & [gwaitfao] & 'Arriero de pico negro' \\
\hline 7. /want $\int \mathrm{aku} /$ & [gwanchaco] & 'Pastorero peruano' \\
\hline 8. /šinku/ & [Singo] & 'Gallinazo de cabeza negra' \\
\hline 9. /turit $\widehat{\int a /}$ & [torit $\widehat{a} \mathrm{a}]$ & 'Cucarachero común' \\
\hline 10. /tuku/ & [tuko] & 'Búho americano' \\
\hline 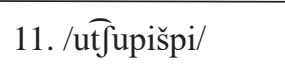 & [gwatfupifpe] & 'Fringilo peruano' \\
\hline 12. /qinti/ & [kinde] & 'Picaflor o colibrí' \\
\hline
\end{tabular}


Como se observa, los fonemas /u/, /i/ del quechua ${ }^{5}$ cambian de punto de articulación al pasar al castellano andino motivados por los contextos fonológicos; por ejemplo, las palabras que terminan en el fonema /u/,/i/ de una lengua originaria cambian a /o/, /e/ en el castellano andino.

El tercer proceso fonológico que se presenta en los nombres de aves del castellano andino de Chota es la despalatalización, que consiste en la pérdida del sonido palatal. De esta manera, los sonidos palatales $/ \lambda / \mathrm{y} / \tilde{\mathrm{n}} /$, al pasar de una lengua originaria al castellano andino, han cambiado a una lateral alveolar $/ 1 / \mathrm{y}$ una nasal alveolar /n/, respectivamente, en el castellano andino. A continuación, se presenta un cuadro con los nombres que sufren estos procesos fonológicos.

\section{Tabla 12.}

La despalatalización en los nombres de aves

Despalatalización de $/ \lambda /, / \tilde{\mathbf{n}} /$

\begin{tabular}{|c|c|c|}
\hline Lengua originaria & Castellano andino & Glosa \\
\hline 1. / $\lambda$ iwin/ & [ligwín] & 'Especie no identificada' \\
\hline 2. /qiw $\lambda \mathrm{a} /$ & [kigwela] & 'Gaviota andina' \\
\hline 3. /pitfuñ/ & [piţfón] & 'Especie no identificada' \\
\hline
\end{tabular}

El cuarto proceso fonológico que han experimentado las palabras de la avifauna de Chota es el reforzamiento que consiste en el refuerzo consonántico que se da solo para [w], [gw] o [rw] al inicio de palabra en el español (Calvo-Shadid, 2008). Así, algunas palabras de las lenguas originarias (quechua) que en ataque silábico inician con la semiconsonante $[\mathrm{w}]$ se refuerzan con una consonante formando $[\mathrm{gw}]$.

\section{Tabla 13.}

El reforzamiento en los nombres de aves

\section{Reforzamiento de /w/}

\begin{tabular}{|l|c|c|}
\hline Lengua originaria & Castellano andino & Glosa \\
\hline 1. /wayt]aw/ & [gwaitfao] & Arriero de pico negro' \\
\hline 2. /want]aku/ & [gwanchaco] & 'Pastorero peruano' \\
\hline 3. /wayanay/ & [gwajana] & 'Golondrina blanquiazul' \\
\hline 4. / $/ \mathrm{iwwin/}$ & [ligwín] & 'Ave no identificada' \\
\hline 5. /ut [upišpi/ & [gwat]upifpe] & 'Fringilo peruano' \\
\hline 6. /qiw $\lambda \mathrm{a} /$ & [kigwela] & 'Gaviota andina' \\
\hline
\end{tabular}


Como se observa, se tiene el proceso de reforzamiento de /w/ en el ataque vocálico de 1 a 6 ; mientras que, en 5 y 6 se tiene la inserción de /a/ y /e/ respectivamente. Ello se da por razones de eufonía y comodidad articulatoria en el español andino. El último proceso fonológico es la elisión, que consiste en la pérdida de un segmento que estaba representando fonológicamente, pero que ya no se representa en el plano fonético (Obediente, 2007). Así, es evidente la elisión de la semiconsonante /y/ de algunos nombres en el castellano andino.

Tabla 14.

La elisión en los nombres de aves

Elisión de /y/

\begin{tabular}{|l|c|c|}
\hline Lengua originaria & Castellano andino & Glosa \\
\hline 1. $/$ t fuqyay/ & {$[\widetilde{\mathrm{t}}$ fukia] } & 'Zorzal chiguanco' \\
\hline 2. /wayanay/ & [gwajana] & 'Golondrina blanquiazul' \\
\hline 3. /liqiliqi/ & {$[$ liklik] } & 'Avefría andina' \\
\hline
\end{tabular}

Regla: $\mathrm{y}>\varnothing /$ \#

\subsection{Concepción y representación de las aves en la cosmovisión andina}

Por último, se ha hecho un análisis etnológico sobre su concepción y representación de las aves. Estas, en la cosmovisión andina, son concebidas a partir de su comportamiento, es decir, las aves que realizan perjuicio en los cultivos son negativas; mientras que las que no afectan los cultivos son concebidas como positivas o, muchas veces, depende de su representación. Por otro lado, no todas las aves, en la cosmovisión andina, tienen una representación simbólica. Por ello, algunas de estas tienden a representar ciertas realidades, como la llegada de la lluvia, la laboriosidad, la prevención del peligro, la plaga para el cultivo, la confrontación entre las personas, el infortunio en el futuro, la visita de un familiar o pariente, y la muerte de una persona. A continuación, presentamos un cuadro que esquematiza lo mencionado. 
Tabla 15.

Concepción y representación de las aves

\section{Concepción}

\begin{tabular}{|l|l|}
\hline Negativas & \multicolumn{1}{|c|}{ Positivas } \\
\hline $\begin{array}{l}\text { Cargacha, chiroca, choroco, hal- } \\
\text { cón, huachupishpe, huaichao, } \\
\text { indiopishgo, liwín, pachatuco, } \\
\text { periquito, pichón, potocha, pugo, } \\
\text { shingo, suekcha, torricha, tuco }\end{array}$ & $\begin{array}{c}\text { Canario, chilala, chinalinda, chotacabras, chuquia, } \\
\text { cuculí, garcita, guardacaballo, guayana, huanchaco, } \\
\text { liclic, mulloshingo, patito, putilla, quienquien, qui- } \\
\text { huela, quinde, tordo, tortolita, turca, zorzalito }\end{array}$ \\
\hline Llegada de la lluvia & Representaciòn \\
\hline Laboriosidad & Cargacha, chuquia, garcita, guayana, liclic, quihuela \\
\hline Prevención del peligro & Chilala \\
\hline Plaga para el cultivo & Chilala, liclic \\
\hline Confrontación entre las personas & Chiroca, indiopishgo, huachupishpe \\
\hline Pereza de las personas & Choroco \\
\hline Infortunio en el futuro & Huaichao, shingo \\
\hline Visita de un familiar o pariente & Quinde \\
\hline Muerte de una persona & Pachatuco, potocha, pugo, shingo, suekcha, tuco \\
\hline
\end{tabular}

\section{Conclusiones}

Una de las conclusiones a las que se ha llegado es que los nombres de la avifauna de Chota evidencian una filiación lingüística de las lenguas quechua, mochica y culle. Asimismo, se ha determinado que un grupo de estos nombres son de procedencia castellana y otro grupo son de origen desconocido.

Otra de las conclusiones es que los nombres de la avifauna de Chota para llegar al uso actual presentan procesos lingüísticos: morfológicos y fonológicos. En el primero los nombres se forman a través de yuxtaposición, reduplicación, derivación y lexicalización. Y en cuanto a lo fonológico, mediante el análisis se ha evidenciado la sonorización, bajamiento vocálico, despalatalización, reforzamiento y elisión.

Por último, partiendo del conocimiento cultural de los entrevistados, las aves del lugar objeto de estudio son concebidas como positivas y negativas. Asimismo, muchas de estas aves tienen una representación simbólica en la cosmovisión de los hablantes de Chota. 


\section{Notas}

1 Alarcón (1991-1992) explica la presencia de culturas preíncas como Pacopampa, la más antigua. A continuación, están Chavín, Chetilla, Tiahuanaco, Chimú y finalmente Inca.

2 El estudio de Andrade-Ciudad (2010) explica la posible convivencia de las lenguas culle, quechua, den y cat en el área de Cajamarca prehispánica; asimismo, no descarta la posibilidad de una toponimia quechumara, es decir, nombres con rasgos de quechua y aimara como Lampadén, Marcadén, Cuscudén, Llamadón, Cascadén, Puchudén y Cochadén, propuestos por Torero en 1989 como toponimia de la lengua hipotética den. En este sentido, otro estudio sobre el panorama actual de la toponimia de Cajamarca (Torres, 2019) evidencia la presencia de topónimos quechua (Marcopata, Andamayo, Atoctambo, Chontabamba), culle (Ushun, Chucumaca, Iraca, Suro, Choloque, Maichil, Muchal), quechua-culle (Chucucerca, Shitacucho, Shitapampa, Inamiaco, Cumpampa), quechua-cat (Cadmalca), cat-desconocido (Huallangate, Susangate, Añalcate, Añicate, Lizcate), den-desconocido (Musaden, Llangodén, Paden) y mochica (Checopon, Pacan Nique), mochica-desconocido (Tuspon, Mazacnique, Yaypon), culle-mochica (Chuchupon) en la provincia de Chota (pp. 251-278). Asimismo, este autor explica que en su análisis toponímico los nombres con rasgos aimara han sido considerados como quechua.

3 Dentro de la teoría de los cambios lingüísticos, el sustrato es la conservación de ciertos sonidos o el uso de construcciones idiomáticas cuando un pueblo abandona su lengua nativa e inicia a hablar otra lengua (Alcaraz y Martínez, 2004).

4 Sistema consonántico del quechua de Cajamarca propuesto por Quesada (2006): oclusivas (labiales: /p/ (/b/), dentalveolares: /t/ (/d/), palatales: /č/ / $/ \mathrm{c} / \mathrm{j} /$, velares: /k/ (/g/), uvulares: /q/), fricativas (labiales: (/f/), dentalveolares: /s/, palatales: /š/ /s/, velares: /x/), nasales (labiales: /m/, dental alveolares: $/ \mathrm{n} /$, palatales: $/ \tilde{\mathrm{n}} /$ ), lateral (dentalveolares: /1/), vibrantes (dentalveolares: $/ \mathrm{r} /$, palatales $/ \check{\mathrm{r}} /$ ), semiconsonantes (labiales: /w/, palatales: /y/).

5 Para Quesada (2006), el sistema vocálico del quechua de Cajamarca es trivocálico, de dos grandes aberturas: alta (anterior /i/ y posterior /u/) y baja (central /a/). Asimismo, enfatiza que a pesar de "la acelerada penetración del español [...] [este dialecto] parece haber rechazo los fonemas /e/ y /o/ de la lengua española”. Sin embargo, solo en ambientes bilingües ha ingresado los dos fonemas aludidos del español: por ejemplo, en los siguientes pares mínimos: “/čoču/ 'abuelo': /čuču/ 'pezón', /mesa/ 'mesa': /misa/ 'misa', /kunka/ 'cuello': /konka, 'obra”' (p. 51). 


\section{Contribución del autor}

Wilmer Burga Muñoz ha participado en la elaboración, la compilación de datos, la redacción y el consentimiento de la versión final del presente artículo.

\section{Fuente de financiamiento}

La investigación es autofinanciada.

\section{Conflictos de interés}

Ninguno.

\section{Trayectoria académica}

Wilmer Burga Muñoz es licenciado en Lenguaje y Literatura por la Universidad Nacional de Cajamarca, con estudios de inglés avanzado en el Instituto Británico de Lima y un diplomado en High Education en LIU (Laureate International Universities). Es egresado de la maestría de Lingüística de la Universidad Nacional Mayor de San Marcos. Ejerce la docencia universitaria en universidades privadas y públicas. Es autor de un artículo literario titulado "Los obstáculos del triunfo" y del cuento "El silencio".

\section{Referencias bibliográficas}

Alcaraz, E. y Martínez, A. (2004). Diccionario de lingüística moderna (2. ${ }^{a}$ ed.). Ariel. Andrade-Ciudad, L. (1999). Topónimos de una lengua extinta en un listado de 1943. Lexis, 23(2), 401-425. http://revistas.pucp.edu.pe/index.php/lexis/ article/view/7240/7445

Andrade-Ciudad, L. (2010). Contactos y fronteras de lenguas en la Cajamarca prehispánica. Boletín de Arqueología PUCP, (14), 165-180. http://revistas.pucp. edu.pe/index.php/boletindearqueologia/article/download/1213/1187/

Andrade-Ciudad, L. (2016). El castellano andino norperuano como una variedad tradicional. Estudios de Lingüistica del Español, (37), 71-86. https://www.raco. cat/index.php/Elies/article/view/327449/417980

Alarcón, P. (1991-1992). Arqueología de Chota. Arquitectura y arte lítico. Festival del Libro Chotano.

Berríos, J. ([1985] 2016). Monografía histórica de Chota. https://es.slideshare.net/cyberespia/monografia-histrica-de-chota-jorge-berrios-alarcon 
Bueno, M. (1994). La onomatopeya y su proceso de lexicalización: notas para un estudio. Anuario de Estudios Filológicos, 17, 15-26. https://dialnet.unirioja.es/ servlet/articulo?codigo $=58804$

Calvo-Shadid, A. (2008). La semiconsonantes y semivocales en los diptongos del español: propuesta de análisis fonológico. Filologia y Lingüística, 34(2), 107142.

Cancino, R. (2019). Denominación de nombres de aves en el quechua de La Unión-Huánuco. [Tesis de maestría, Universidad Nacional Mayor de San Marcos]. https://hdl.handle.net/20.500.12672/11553

Casado, M. (1991). Lenguaje y cultura: la etnolingüística. Síntesis.

Cerna, L. (2015). La motivación y el fonosimbolismo, características del signo lingüístico del idioma quechua de Cajamarca. Huella Docente, 1(1), 8-19.

Cerrón-Palomino, P. (1989). Quechua y mochica: lenguas en contacto. Lexis, 13(1), 47-68. http://revistas.pucp.edu.pe/index.php/lexis/article/view/5371/5370

Cruz-Villegas, J. (1982). Catacaos. Origen y evolución. Historia de Catacaos. Centro de Investigación y Promoción del Campesinado (Cipca).

Escandell, M. (2014). La estructura de las palabras. En Fundación Ramón Areces (eds.) Claves del lenguaje bumano (pp.121-153). Universitaria Ramón Areces.

Flores, M. (2012). La lengua culle, entrevista a Manuel Flores Reyna por Danilo Sánchez Lihón. http://gonzaloespino.blogspot.com/2012/05/la-lengua-culle-entrevista-manuel.html

Isbell, W. (2010). La arqueología wari y la dispersión del quechua. Boletín de Arqueologia PUCP, (14), 199-220.

Itier, C. (2013). Las bases geográficas de la lengua vehicular del imperio inca. Bulletin de l'Institut Français d'Études Andines, 42(2), 237-260. https://doi. org/10.4000/bifea.8030

Macera, P., Jiménez, A. y Franke, I. (1997). Trujillo del Perú. Baltazar Jaime Martínez Compañón. Acuarelas. Siglo XVIII. Ausonia.

Ministerio de Educación. (2018). Lenguas originarias del Perú. http://repositorio.minedu.gob.pe/handle/MINEDU/6261

Obediente, E. (2007). Fonética y fonología (3ra. ed.). Consejo de Publicaciones de la Universidad de los Andes. 
Pérez, J. (2016). La representación de los procesos fonológicos: a propósito del descenso vocálico del quechua. Revista del Instituto Riva-Agüero, 1(1), 77-124. https://dialnet.unirioja.es/descarga/articulo/6135767.pdf

Puig-Tarrats, E. (2007). Breve diccionario folclórico piurano. Universidad de Piura.

Quesada, F. (1976). Diccionario quechua: cajamarca-cañaris. Ministerio de Educación.

Quesada, F. (2006). Quechua de Cajamarca. Mantaro.

Rosas, H. y Shady, R. (2017). Pacopampa: Un centro formativo en la sierra norperuana. ISHRA, Revista del Instituto Seminario de Historia Rural Andina, 2(3), 145-157. http://dx.doi.org/10.15381/ishra.v2i3.14820

Salas, J. (2002). Diccionario mochica-castellano castellano-mochica. Universidad de San Martín de Porres.

Salas, J. (2012). Etimologías. Lima: Academia Peruana de la Lengua

Siegel, J. (2018). Demographic, and Socioeconomic Basis of Ethnolinguistics. Springer.

Torero, A. (2002). Idiomas de los Andes. Lingüistica e historia. Horizonte.

Torres, D. (2019). Panorama lingüístico del departamento de Cajamarca a partir del examen de la toponimia actual. [Tesis de maestría, Pontificia Universidad Católica del Perú]. http://hdl.handle.net/20.500.12404/15199

Ullman, S. (1972). Semántica. Introducción a la ciencia del significado. Aguilar.

Varela, S. (2018). Morfología léxica: la formación de palabras. Gredos.

Watanabe, S. (2001). Wari y Cajamarca. Boletin de Arqueología PUCP, (5), 531-541. http://revistas.pucp.edu.pe/index.php/boletindearqueologia/article/down$\operatorname{load} / 2524 / 2468 /$ 